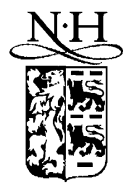

ELSEVIER
Available online at www.sciencedirect.com

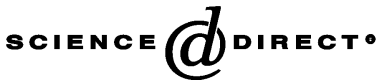

Nuclear Instruments and Methods in Physics Research A 503 (2003) 322-327

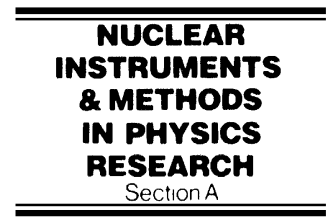

www.elsevier.com/locate/nima

\title{
Design and particle tracking of FFAG
}

\author{
S. Machida
}

KEK, 1-1 Oho, Tsukuba-shi, Ibaraki-ken 305-0801, Japan

\begin{abstract}
Modeling FFAG optics using tools for ordinary synchrotrons is described. Some tracking results are also presented. (C) 2003 Elsevier Science B.V. All rights reserved.
\end{abstract}

PACS: 29.27.Eg; 41.75.Lx

Keywords: FFAG (Fixed Field Alternating Gradient); Optics; Code

\section{Introduction}

Fixed Field Alternating Gradient (FFAG) synchrotrons are invented in 1953 right after the principle of the alternating gradient focusing [1]. At that time, machine parameters are calculated analytically. Complicated equations and approximations appear in old papers. It is, however, not necessary to strictly follow those formalism to design FFAG now. Design tools for an ordinary synchrotron developed for the last 30 years can be applied to FFAG. Sophisticated modern particle tracking is also possible in FFAG using codes developed recently.

\section{Optics design of FFAG}

Although FFAG magnet has strong nonlinearity as Eq. (1) indicates, it should be still a good approximation of assuming linear focusing force

E-mail address: shinji.machida@kek.jp (S. Machida). within the small oscillation amplitude.

$B(r)=B\left(r_{0}\right)\left(\frac{r}{r_{0}}\right)^{k}$

where $B\left(r_{0}\right)$ is the reference field strength at $r=r_{0}$ and $k$ is called the field index. Once we ignore the nonlinear terms, the optics design tool for an ordinary synchrotron will calculate the orbit and focusing property such as tune and lattice functions. Still, one significant difference from an ordinary synchrotron is that it is not easy to find a closed orbit. Furthermore, it depends on momentum.

In the following, we will explain three different cases of the radial sector FFAG, namely, singlet focusing lattice, two beam accelerator lattice as a variation of the singlet lattice, and triplet focusing lattice.

\subsection{Singlet focusing lattice}

A radial sector FFAG with singlet focusing lattice consists of a normal bending magnet named 
as F-bend and a reverse bending magnet named as D-bend. Although the orbit does not have the constant curvature inside each magnet because of the field function of Eq. (1), we assume an arc with constant curvature as shown in Fig. 1. The figure shows a half sector starting from the center of F-bend to the center of D-bend.

Here, $\beta_{\mathrm{F}}$ is a half of open angle of $\mathrm{F}$-bend, $\theta_{\mathrm{F}}$ is bending radius of F-bend, $\rho_{\mathrm{F}}$ is bending radius of $\mathrm{F}$ bend, and $L_{\mathrm{F}}$ is path length of F-bend. Each symbol with $\mathrm{D}$ means that of D-bend. The $r_{0}, r_{1}, r_{2}$, and $r_{3}$ are the orbit radius at each azimuthal position.

Now, let us find relations among those parameters. Assume that a whole ring consists of $N$ sectors, and $\beta_{\mathrm{F}}, \beta_{\mathrm{D}}$, and $\theta_{\mathrm{F}}$ are given. Then, the other parameters are scaled to $r_{0}$ as

$$
\frac{\rho_{\mathrm{F}}}{r_{0}}=\frac{\tan \beta_{\mathrm{F}}}{\sin \theta_{\mathrm{F}}+\left(1-\cos \theta_{\mathrm{F}}\right) \tan \beta_{\mathrm{F}}}
$$

$$
\frac{\rho_{\mathrm{D}}}{r_{2}}=\frac{\sin \beta_{\mathrm{D}}}{\sin \theta_{\mathrm{D}}}
$$

$$
\begin{aligned}
\frac{r_{2}}{r_{0}}= & {\left[1-\frac{\rho_{\mathrm{F}}}{r_{0}}\left(1-\frac{1}{\sin \left(\pi-\theta_{\mathrm{F}}\right)}\right)\right] } \\
& \times \frac{\tan \left(\pi-\theta_{\mathrm{F}}\right)}{\cos \left(\pi / N-\beta_{\mathrm{D}}\right)\left[\tan \left(\pi / N-\beta_{\mathrm{D}}\right)+\tan \left(\pi-\theta_{\mathrm{F}}\right)\right]}
\end{aligned}
$$

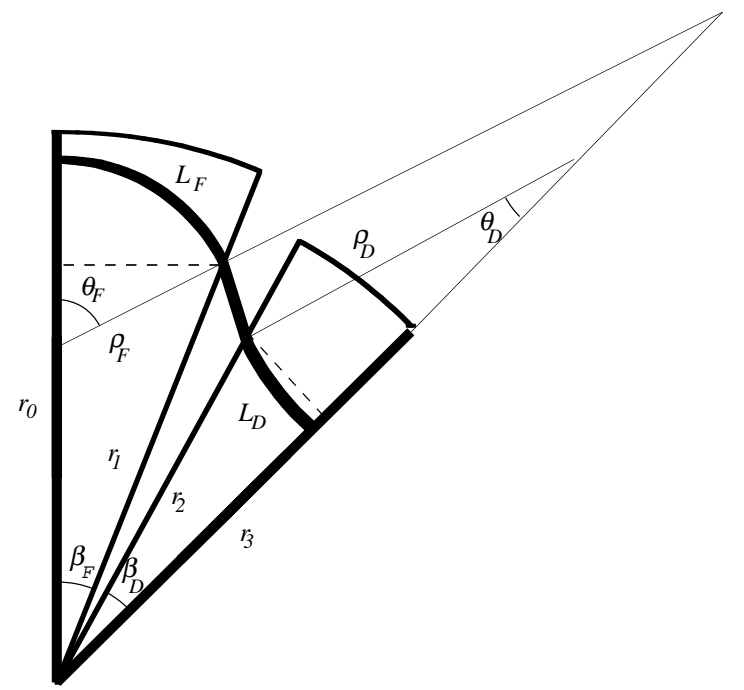

Fig. 1. Model of FFAG with singlet focusing lattice.



Fig. 2. Footprint of a singlet focusing FFAG.

$r_{1}=\frac{\rho_{\mathrm{F}} \sin \theta_{\mathrm{F}}}{\sin \beta_{\mathrm{F}}}$.

The edge angle at the both edges of F-and D-bend are

$\varepsilon_{\mathrm{F}}=\frac{\theta_{\mathrm{F}}-\beta_{\mathrm{F}}}{2 \theta_{\mathrm{F}}}$

$\varepsilon_{\mathrm{D}}=\frac{\theta_{\mathrm{D}}+\beta_{\mathrm{D}}}{\theta_{\mathrm{D}}}$.

It is ready to model FFAG with a design tool for an ordinary synchrotron such as SAD and MAD. As an example, the following parameters are given. That is, $N=8, \beta_{\mathrm{F}}=8.6^{\circ}, \theta_{\mathrm{F}}=$ $29.6^{\circ}, \beta_{\mathrm{D}}=5.7^{\circ}, \theta_{\mathrm{D}}=180 / N-\theta_{\mathrm{F}}^{\circ}$, and $k=2.5$. SAD result shows that the horizontal phase advance per sector is $100^{\circ}$ and the vertical one is $57^{\circ}$. The footprint and lattice functions are shown in Figs. 2 and 3, respectively.

\subsection{Two beam accelerator}

It is interesting that the particles with the same sign can rotate in opposite direction if the F- and D-bend of the singlet focusing are identical except the sign of the field. As a result, a collider in a single FFAG can be realized. That is due to the fact that the orbit of radial sector FFAG tends to 


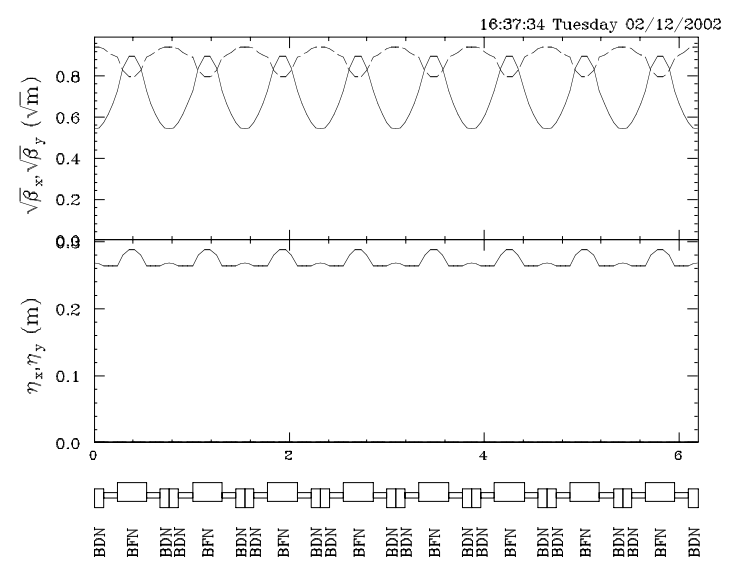

Fig. 3. Lattice functions calculated with SAD for a singlet focusing FFAG.

be inner at D-bend and outer at F-bend. Even though the field strength is given by Eq. (1) for the both magnets, the bending angle at F-bend is always larger than that at D-bend and the orbit makes a ring. For a particle going in the other direction, F-bend acts as D-bend and vice versa.

In order to model the two beam accelerator, the following conditions should be added to the singlet focusing lattice. The first one is obvious and the second is the approximation, that is, ratio of the bending angle is determined by the orbit radius at the center of $\mathrm{F}$ - and $\mathrm{D}$-bend, as well as by the path length at the F- and D-bend, which we assume to be proportional to the orbit radius.

$\beta_{\mathrm{F}}=\beta_{\mathrm{D}}$

$\frac{\theta_{\mathrm{F}}}{\theta_{\mathrm{D}}}=\left(\frac{r_{0}}{r_{3}}\right)^{k+1}$.

In practice, $\theta_{\mathrm{F}}$ is determined by iteration given $\beta_{\mathrm{F}}$ and $\beta_{\mathrm{D}}$. As an example, the following parameters are considered. That is, $N=16, \beta_{\mathrm{F}}=\beta_{\mathrm{D}}=$ $1.7^{\circ}, \theta_{\mathrm{F}}=26^{\circ}, \quad \theta_{\mathrm{D}}=180 / N-\theta_{\mathrm{F}}^{\circ}, \quad$ and $k=8$. The phase advance per sector is $124^{\circ}$ and $156^{\circ}$ for the horizontal and vertical, respectively. The footprint and lattice functions are shown in Figs. 4 and 5 .



Fig. 4. Footprint of a two-beam accelerator.

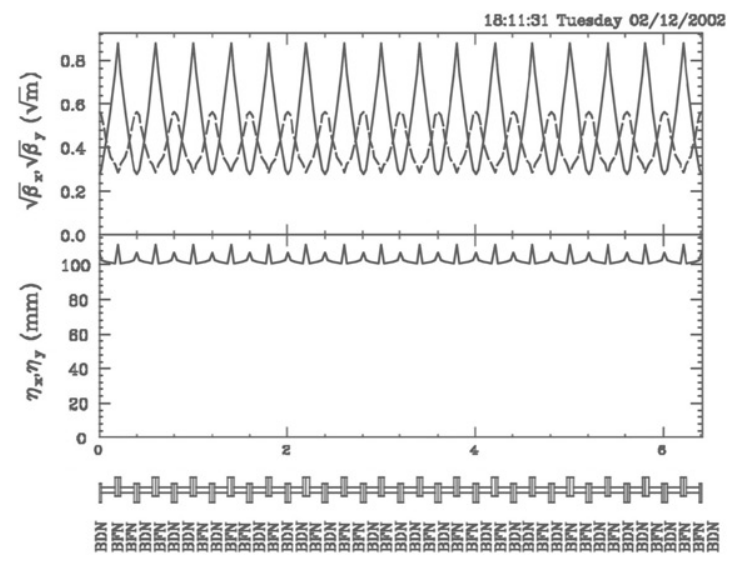

Fig. 5. Lattice functions of a two-beam accelerator.

\subsection{Triplet focusing lattice}

The last example is an FFAG lattice with triplet focusing. The triplet focusing lattice can be made by splitting D-bend into two and adding them to the adjacent F-bends. In that way, the number of drift space between F- and D-bend becomes a half, whereas the length of the drift space is doubled. That is advantageous to install injection and extraction hardware. Another advantage is the well-shaped field distribution. The fringe field between F-bend and D-bend is shapely terminated because of the cancellation of the opposite fields. 




Fig. 6. Half sector of a triplet lattice starting from the center of F-bend to the center of drift space.

In order to model a triplet focusing lattice, magnet position and orbit are assumed as shown in Fig. 6.

The parameter dependence is slightly different from the singlet focusing. That is

$\frac{\rho_{\mathrm{F}}}{r_{0}}=\frac{\tan \beta_{\mathrm{F}}}{\sin \theta_{\mathrm{F}}+\left(1-\cos \theta_{\mathrm{F}}\right) \tan \beta_{\mathrm{F}}}$

$r_{1}=\frac{\rho_{\mathrm{F}} \sin \theta_{\mathrm{F}}}{\sin \beta_{\mathrm{F}}}$

$\frac{\rho_{\mathrm{D}}}{\rho_{\mathrm{F}}}=\frac{\sin \theta_{\mathrm{F}}}{\sin \beta_{\mathrm{F}}}\left[\frac{\sin \left(\pi / N-\beta_{\mathrm{F}}\right)-\cos \left(\pi / N-\beta_{\mathrm{F}}\right) \tan \left(\pi / N-\beta_{\mathrm{F}}-\beta_{\mathrm{D}}\right)}{\sin \left(\theta_{\mathrm{F}}-\pi / N\right)-\left[1-\cos \left(\theta_{\mathrm{F}}-\pi / N\right)\right] \tan \left(\pi / N-\beta_{\mathrm{F}}-\beta_{\mathrm{D}}\right)}\right]$.

The edge angle at the entrance and exit of Dbend is different. One to F-bend side is

$\varepsilon_{\mathrm{D}, \mathrm{F}}=\frac{\theta_{\mathrm{F}}-\beta_{\mathrm{F}}}{\theta_{\mathrm{D}}}$

and the other is

$\varepsilon_{\mathrm{D}, \mathrm{O}}=-\frac{\pi / N-\beta_{\mathrm{F}}-\beta_{\mathrm{F}}}{\theta_{\mathrm{D}}}$.

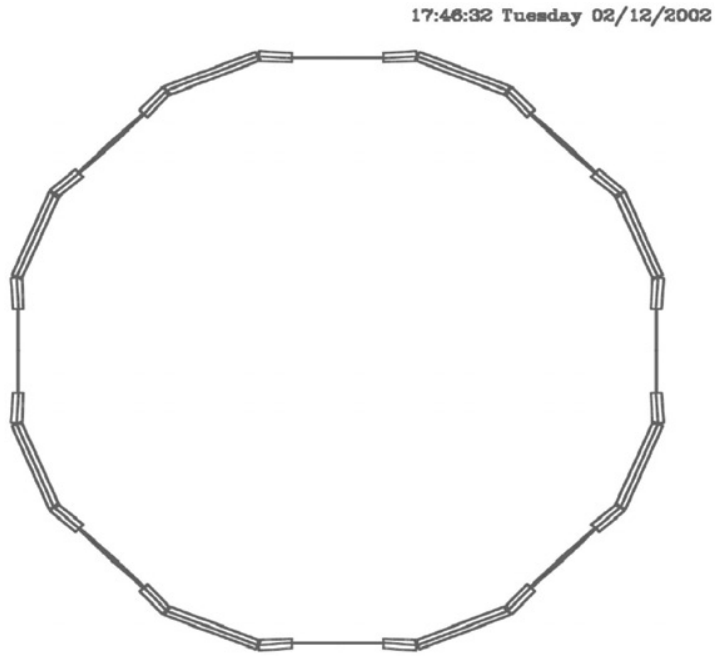

Fig. 7. Footprint of a triplet focusing FFAG.

The both sides of F-bend has the same angle as

$\varepsilon_{\mathrm{F}}=\frac{\theta_{\mathrm{F}}-\beta_{\mathrm{F}}}{2 \theta_{\mathrm{F}}}$

As an example, the following parameters similar to the singlet lattice are considered. That is, $N=8$, $\beta_{\mathrm{F}}=8.6^{\circ}, \theta_{\mathrm{F}}=29^{\circ}, \quad \beta_{\mathrm{D}}=5.7^{\circ}, \theta_{\mathrm{D}}=180 / N-$ $\theta_{\mathrm{F}}^{\circ}$, and $k=2.5$. The phase advance per sector is $95^{\circ}$ and $57^{\circ}$ for the horizontal and vertical, respectively. The footprint and lattice functions are shown in Figs. 7 and 8.

\section{Particle tracking of FFAG}

A linearized model we explained is good enough to determine size of a machine and major machine parameters such as tune and beam size. The lattice of the FFAG, however, is full of nonlinearity, and particle tracking is eventually necessary to know the behavior of large-amplitude particles and 


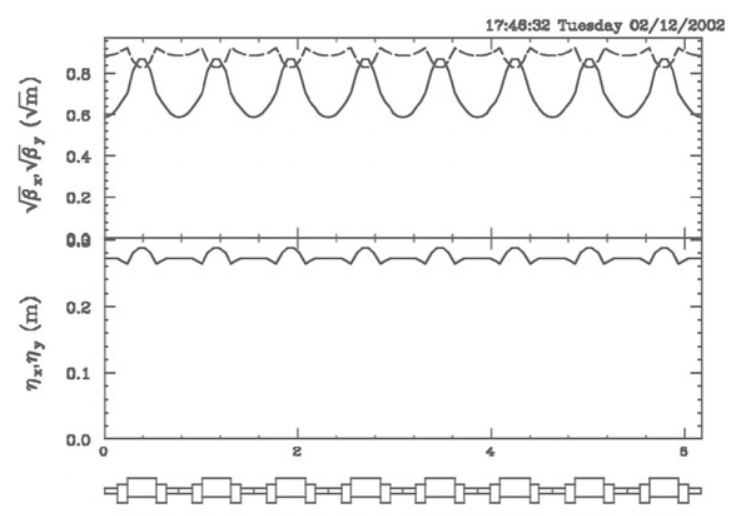

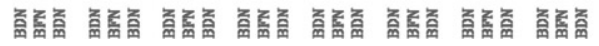

Fig. 8. Lattice functions of a triplet focusing FFAG.



Fig. 9. Poincare map for several particles with different initial amplitudes.

dynamic aperture. Furthermore, the fringe field of the magnets tends to be large. Particle tracking based on 3D field distribution is required.

A brute force tracking which employs 3D field data on spatial grids and Runge-Kutta integrator is one method. Fig. 9 shows the horizontal Poincare map for several initial amplitudes for the $150 \mathrm{MeV}$ FFAG under construction at KEK. The kinetic energy is fixed at $27.4 \mathrm{MeV}$ and the operating tune is $(3.80,1.19)$. Since $v_{x}=4$ is the third-order structure resonance, strong resonance structure in phase space appears. The amplitude dependent tune is plotted in Fig. 10 by analyzing the data with FFT.

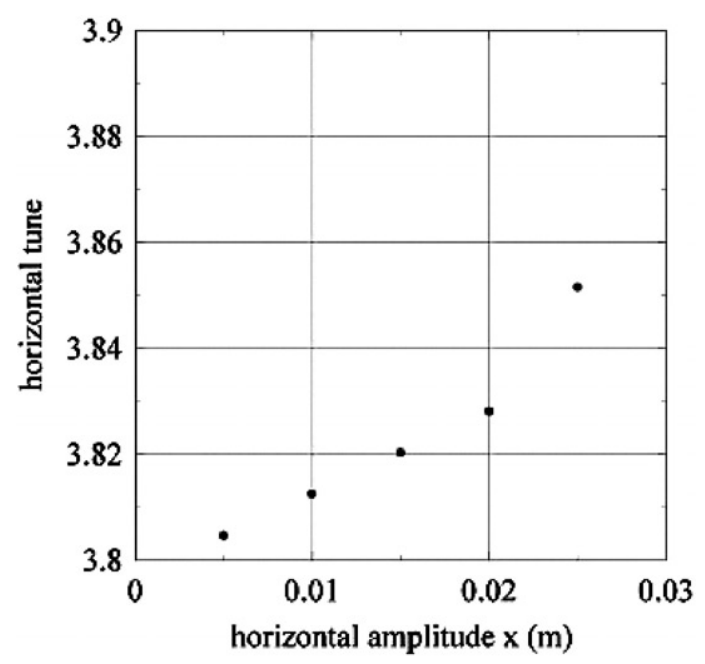

Fig. 10. Amplitude dependence of horizontal tune.

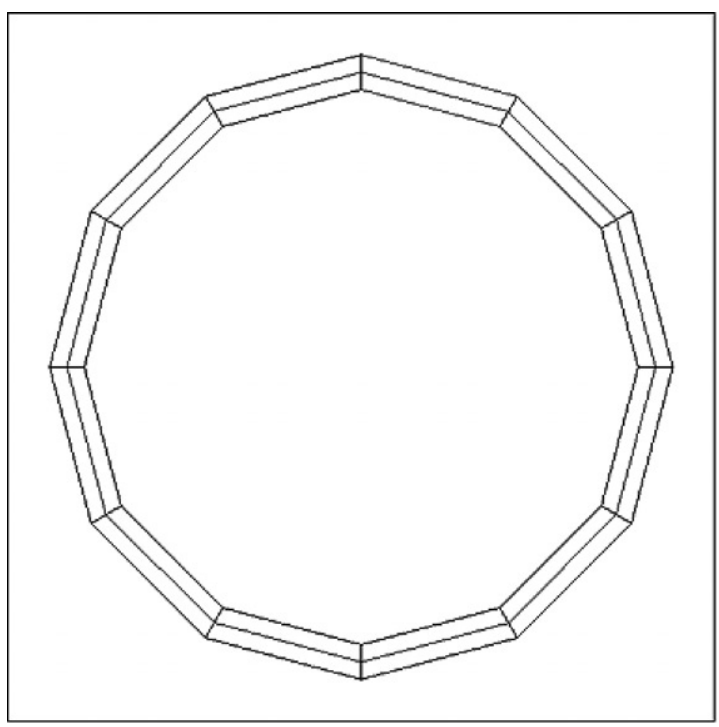

Fig. 11. FFAG with 12 sector is modeled with PTC. A trapezoid including F-, D-bend, and drift space is divided into many thin elements.

More sophisticated tracking can be possible with PTC (Polymorphic Tracking Code) package recently developed by Forest [2]. Instead of interpolating the 3D field data from adjacent grids every time step, we first replace the entire 3D field mapping of one period with a bunch of thin elements aligned in longitudinal direction. In each 


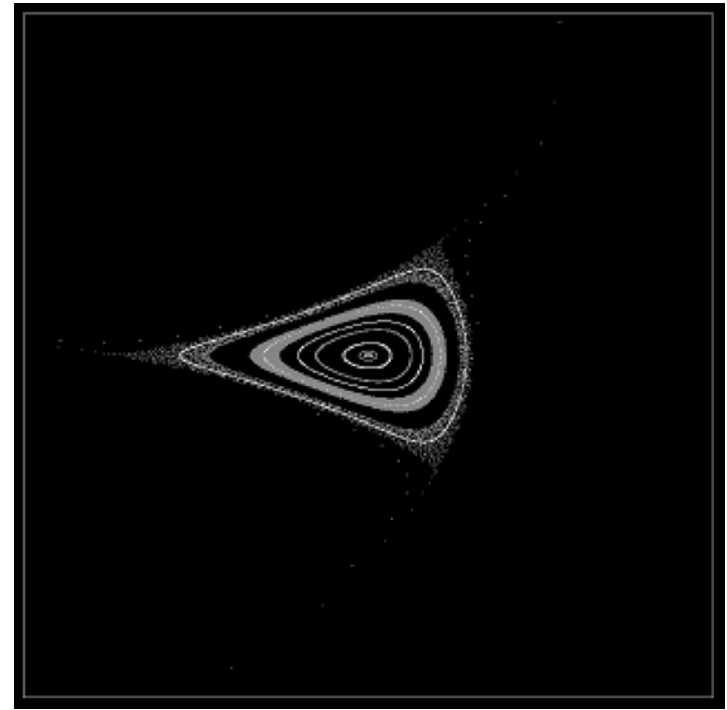

Fig. 12. Tracking results from PTC calculation.

thin element, three magnetic field components are fitted either globally or locally so that its derivatives with respect to the coordinates exist to the necessary order. Once the 3D fields are represented in that manner, real or Taylor type can be integrated.
As an example, $150 \mathrm{MeV}$ FFAG with 12 sector is modeled in the following. Fig. 11 shows a ring with the 12 identical sector. A trapezoid is divided into many thin elements based on the calculation of 3D magnetic fields including F-bend, D-bend, and drift space, all together. Fig. 12 shows tracking result of PTC with the same machine parameters as Fig. 9. In that example, the phasespace behavior and dynamic aperture calculated by the brute force tracking and PTC turns out not so different from each other although the CPU time for PTC tracking is faster.

\section{Acknowledgements}

We would like to thank Prof. E. Forest for his help to use PTC package.

\section{References}

[1] T. Ohkawa, Bull. Phys. Soc. Jpn. (1953); F.T. Cole, R.O. Haxby, L.W. Jones, C.H. Pruett, K.M. Terwilliger, Rev. Sci. Instr. 28 (6) (1957) 403.

[2] E. Forest, F. Schmidt, E. Mcintosh, KEK Report 2002-3, 2002. 